Musées, Patrimoine et Culture scientifiques et techniques

177 | 2018

mai-juin 2018

\title{
Penser avec ses mains ou la philosophie du tinkering
}

Anik Meunier, Brigitte Belleville et Olivier Grant

\section{OpenEdition \\ Journals}

Édition électronique

URL : http://journals.openedition.org/ocim/2557

DOI : $10.4000 /$ ocim. 2557

ISSN : 2108-646X

Éditeur

OCIM

Édition imprimée

Date de publication : 1 mai 2018

Pagination : 29-35

ISSN : 0994-1908

Référence électronique

Anik Meunier, Brigitte Belleville et Olivier Grant, « Penser avec ses mains ou la philosophie du tinkering ", La Lettre de I'OCIM [En ligne], 177 | 2018, mis en ligne le 01 mai 2019, consulté le 05 juin 2019. URL http://journals.openedition.org/ocim/2557 ; DOI : 10.4000/ocim.2557

Ce document a été généré automatiquement le 5 juin 2019.

Tous droits réservés 


\section{Penser avec ses mains ou la philosophie du tinkering}

Anik Meunier, Brigitte Belleville et Olivier Grant

Signature de l'exposition Fabrik au Centre des sciences de Montréal

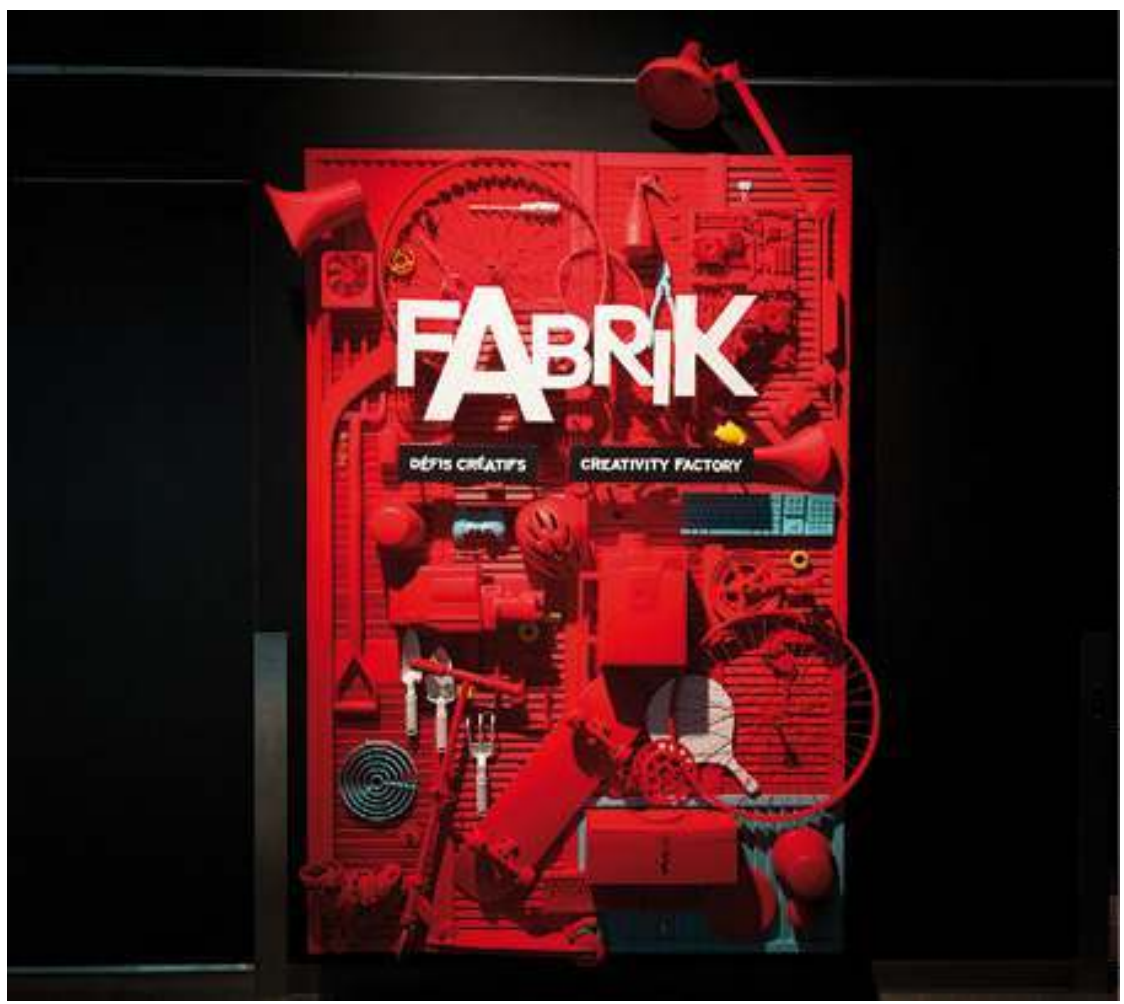

(c) CSM

"L'idéal n'est pas de cumuler ses connaissances mais de développer ses capacités".

John Dewey 
3 En contexte nord américain, on observe des liens de plus en plus soutenus entre les écoles, les musées et les centres de sciences dans le développement de propositions susceptibles de soutenir l'éducation technoscientifique. Plus largement, ces propositions concernent aussi la culture scientifique en contexte d'éducation non formelle et peuvent bénéficier aux citoyens. À ce titre, l'exposition Fabrik du Centre des sciences de Montréal (CSM) a été inspirée d'ateliers de tinkering observés à l'Exploratorium de San Francisco. Fabrik a connu un tel succès qu'elle a été "exportée" à Bergen, en Norvège et a donné naissance à l'exposition Skaperhagen ou The Creative Garden au centre des sciences Vilvite.

\section{La culture scientifique et technologique autrement}

4 L'acquisition des éléments essentiels de la culture scientifique et technologique repose sur la pratique de démarches scientifiques dès le plus jeune âge et tout au long de la vie. Il est largement admis que l'école n'est plus le seul lieu légitime de la diffusion de ces savoirs et que d'autres contextes contribuent à développer la conscience du monde qui nous entoure. En effet, un ensemble d'équipements à caractère scientifique et technique permettent de se construire une représentation globale et cohérente du monde et de mieux comprendre son environnement quotidien. Comprise comme une dimension fondamentale de la formation des élèves, la culture scientifique et technologique repose ainsi sur une connaissance des principes et des finalités du raisonnement scientifique, mais aussi sur une pratique effective de démarches d'investigation et de résolution de problèmes scientifiques. Dans ce contexte, l'école est appelée à transformer ses pratiques, en s'éloignant de l'approche de transmission de connaissances, pour offrir plutôt aux élèves des occasions de développer la capacité d'exploiter efficacement les ressources de la société éducative (Unesco, 2005).

En éducation scientifique, l'école peut mettre à profit les musées ou les centres de sciences pour susciter des apprentissages contextualisés à propos des sciences et des connaissances qu'elles produisent. Les lieux extra scolaires jouissent d'un certain statut d'autorité en ce qui concerne la diffusion de connaissances scientifiques validées. Ils sont des lieux privilégiés d'apprentissage autonome à propos des sciences et de la technologie et l'école a tout avantage à les exploiter. Le Centre des sciences de Montréal (CSM) dans la foulée du récent renouvellement de ses espaces permanents a conçu et produit une exposition qui prend appui sur le tinkering, une approche originale et innovante dans le développement de la culture scientifique et technologique. Sous la forme d'expositionateliers, les espaces de tinkering visent à développer chez les visiteurs la créativité et le sens de l'innovation par des défis axés sur la manipulation, l'assemblage d'objets et de matériaux avec de vrais outils. Cette proposition du CSM cherche à offrir à ses visiteurs une expérience complémentaire unique.

\section{La démarche de conception : les sources d'inspiration}

6 Des médiations observées actuellement au sein des musées et des expositions questionnent les approches éducatives traditionnelles et les modèles qui y étaient associés. 
L'exposition Fabrik, un espace adapté aux ateliers de groupes.

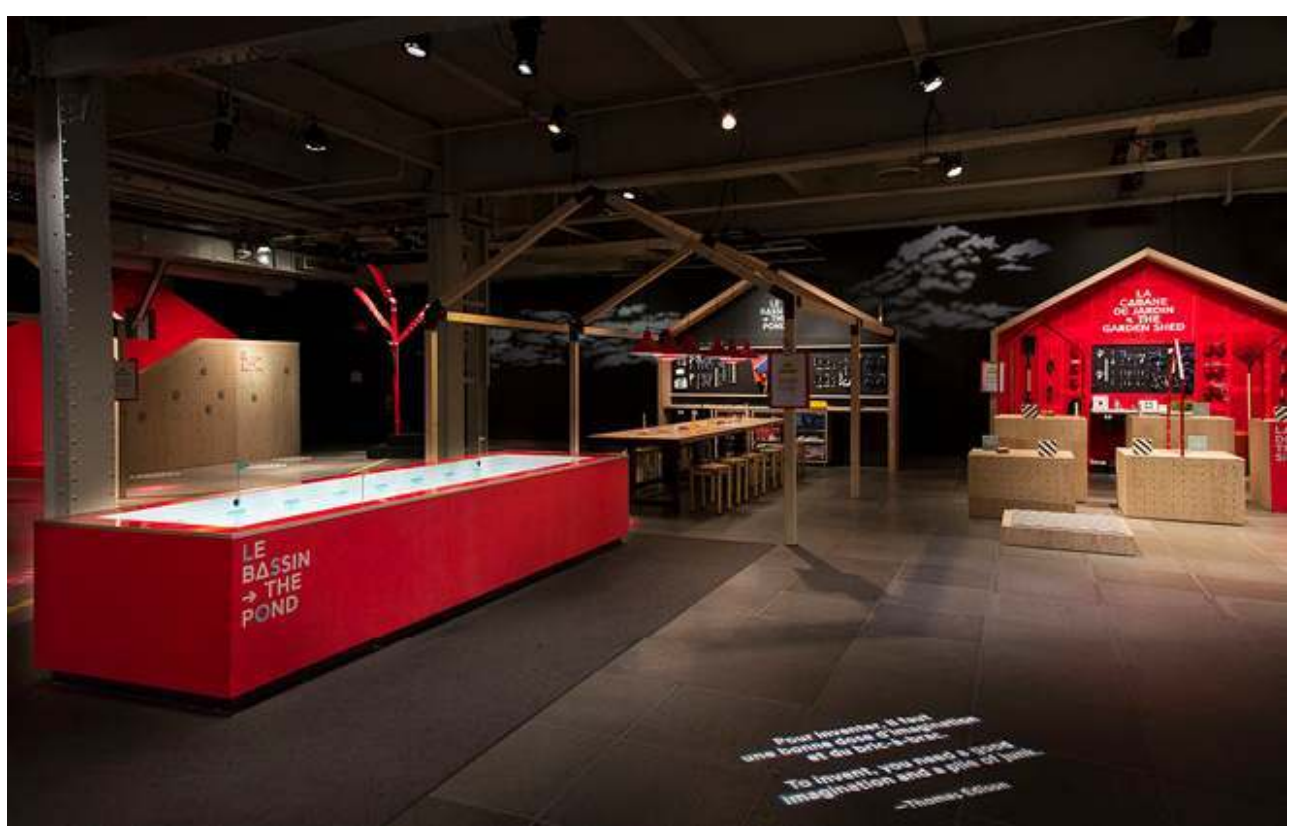

(c) CSM

Fab Lab (ou atelier de fabrication) lieu où l'on peut fabriquer des objets utiles. Le processus se fait habituellement de l'ordinateur via un logiciel de conception à des outils spécialisés comme l'imprimante 3D.

Maker Space lieu où l'on réalise quelque chose de spécifique avec du matériel nouveau et familier. Dans le Maker Space, le fait de construire quelque chose est instructif. La démarche s'inscrit dans le mouvement des DIY - Do it Yourself - où l'on peut construire soi-même un objet en suivant des étapes simples ou un plan précis.

Tinkering Lab lieu qui nécessite un état d'esprit créatif. Il comporte une façon amusante d'approcher et de résoudre des défis par l'expérimentation et une démarche impliquant essais et erreurs. Il favorise ainsi le développement de la pensée créative et donne lieu à de multiples solutions.

En effet, les Fab Labs (faire/défaire/refaire) proposent d'apprendre tout en faisant ( learning by doing de Dewey) et adoptent la philosophie OpenSource (mise à disposition gratuite des contenus). Les ateliers de Tinkering (bricoler/rafistoler/ajuster) introduits par certains centres de sciences mettent de l'avant l'imagination et l'inventivité des visiteurs pour expérimenter des alternatives afin de trouver des solutions à des défis technologiques ou scientifiques. En outre, ces propositions pédagogiques pensent autrement l'apprentissage. Elles adoptent notamment des approches de cocréation et bousculent les modèles d'éducation et d'apprentissage bien ancrés. L'exposition Fabrik s'inspire des Tinkering Labs, expression traduite littéralement par "labos de bidouillage" et s'inscrit dans une tendance de plus en plus répandue ces dernières années, en Amérique $\mathrm{du}$ Nord et en Europe. Ces labos ont notamment été initiés par l'Exploratorium de San Francisco (fondé en 1969) et portent entre autres les noms de Tinkering Studio, Design Lab, Challenge Zone. On en dénombre déjà des dizaines. Chacun détient ses particularités mais 
ils proposent une expérience similaire : mettre à la disposition des visiteurs des matériaux et des outils simples, et les inviter à réaliser des activités manuelles plus ou moins structurées, afin de développer leur créativité. Cette approche fait écho à plusieurs innovations culturelles et sociales, notamment celle des FabLabs, concept né en 2002 au Massachussetts Institute of Technology (MIT, Boston). Les FabLabs, contraction de l'anglais Fabrication Laboratory, "laboratoire de fabrication", sont des lieux où le public a accès à des équipements de fabrication numérique (découpeuse laser, imprimante 3D, fraiseuse CNC et autres) afin de démocratiser les moyens de production et de redonner aux citoyens le pouvoir de fabriquer, de construire, d'innover.

\section{Une muséographie pour relever des défis}

7 L'exposition du CSM se présente comme un grand atelier. L'espace occupé par Fabrik (500 $\mathrm{m}^{2}$ ) propose un aménagement ouvert visant à favoriser la communication, voire la contamination croisée des idées, la pollinisation de notions et de stratégies entre les visiteurs. L'exposition est découpée en plusieurs zones de travail - communément appelées sheds ou cabanons - pour s'approvisionner en matériel, concevoir, fabriquer, expérimenter. Pour le CSM, grâce à cette plateforme, les six espaces de "défis" peuvent être renouvelés facilement au fil des ans. À l'étape de sa conception, une attention particulière a été portée à la modularité, la facilité de montage et le rangement. Fabrik est surtout une plateforme qui contient des équipements uniques, des bancs d'essai flexibles, pour tester les réalisations des visiteurs. Les objectifs de l'exposition consistent à : faire vivre le processus de création, essentiel dans plusieurs domaines scientifiques, technologiques et artistiques ; mener le visiteur à réaliser son potentiel créatif et concevoir l'erreur comme nécessaire et utile. Les messages de l'exposition proposent que le processus de création aide à trouver des solutions originales, que tous peuvent être créatifs et que l'erreur est utile. La capacité d'accueil de l'espace est d'une centaine de personnes et le temps généralement passé dans l'exposition est environ une heure. La médiation active implique la présence de plusieurs éducateurs durant les heures d'opération de l'exposition Fabrik.

\section{Des défis diversifiés et inusités}

Les stations de l'exposition proposent aux visiteurs de relever des défis inusités. Ceux-ci sont axés sur la manipulation de matériaux, d'outils et de technologies. Les défis permettent aux participants de s'initier au processus de création et les encouragent à développer leur pensée et rechercher de nouvelles solutions. En tout temps, un médiateur est présent dans l'exposition. Ce dernier aide les visiteurs en les questionnant ou les guidant plutôt qu'en leur donnant des réponses ou des solutions. Son objectif est de déclencher l'étincelle et la curiosité des usagers. Des matériaux variés sont mis à disposition. Ceux-ci sont souvent employés de manière originale. Ils sont assemblés pour réaliser les défis, puis démontés afin d'être réutilisés par les prochains visiteurs. Le CSM vise des sources d'approvisionnement locales et la réutilisation de rebus d'entreprises commerciales et/ou industrielles. Quant aux outils, des pinces, des ciseaux, des marteaux et des tournevis sont proposés, même aux enfants, puisque pour résoudre de "vrais" défis, il faut utiliser de "vrais" outils. 
Dans Fabrik, des matériaux très divers sont mis à disposition des visiteurs.

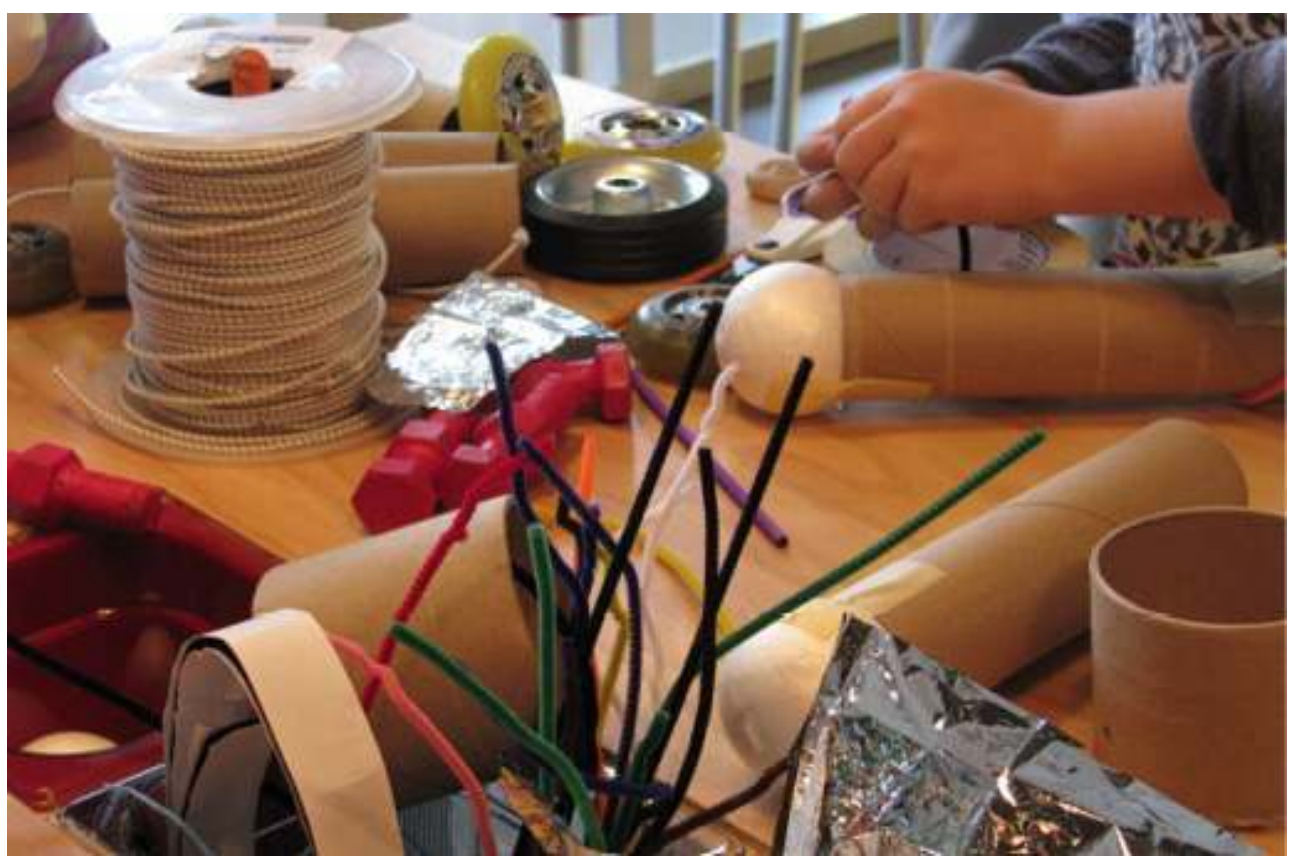

(c) CSM

\section{Les défis créatifs de Fabrik}

- La ruelle

Fabrique un engin pouvant transporter une charge de $25 \mathrm{~g}$ à l'aide du vent : ajoute plus de poids; franchis le fil d'arrivée plus vite; n'utilise pas de roue.

- La cabane de jardin

Conçois une chaîne de réactions qui se déclenchent les unes après les autres : augmente le nombre de réactions; varie les types de réactions (levier, balancier, série de dominos, etc.); joins tes réactions à celles d'autres visiteurs; crée une finale du tonnerre!

- Le hangar

Fabrique une structure lumineuse originale afin d'illuminer le hangar : ajoute des effets de couleurs ; introduis des jeux d'ombre ; ajoute du mouvement.

- Le bassin

Fabrique un engin pouvant transporter une charge de $25 \mathrm{~g}$ d'une rive à l'autre ou crée une structure flottante pouvant supporter une charge de $250 \mathrm{~g}$ : ajoute plus de poids; fais traverser la charge dans le sens de la longueur; fais un aller-retour.

- Le balcon

Trouve un moyen pour que l'objet arrive intact au sol : utilise le moins de matériaux possible ; trouve une solution différente. 
- La corde à linge

Fabrique un engin pouvant se déplacer le long des câbles : déplace une charge de $25 \mathrm{~g}$; ajoute plus de poids ; franchis la distance plus vite.

Un bassin d'eau fait partie d'un des bancs d'essai proposé aux visiteurs. Ces derniers sont invités à faire flotter une masse de $250 \mathrm{~g}$. Derrière ce défi se cachent des notions liées à la flottabilité, la poussée d'Archimède et la masse volumique. Mais, si tous ces savoirs scientifiques ne sont que rarement évoqués, ils demeurent sous-jacents aux défis et aux solutions apportées par les visiteurs et ne sont que rarement abordés par les médiateurs. L'important est d'être créatif et de trouver des solutions pour que la masse ne coule pas au fond du bassin.

\section{Des visiteurs créatifs et actifs}

De tout temps, les êtres humains ont cherché à innover, à créer de nouvelles solutions, que ce soit par nécessité pour faciliter leur quotidien ou même s'approcher un peu plus d'un monde rêvé, atteindre l'inatteignable. L'expérience du visiteur proposée par Fabrik est justement d'inventer. Il entre dans la peau d'un créateur, qu'il soit ingénieur, inventeur ou artiste pour réaliser sa propre "machine, invention, œuvre". Il s'approprie des notions en sciences et en technologies qui l'aident à faire des choix tout en suivant un processus de création. Fabrik accueille des visiteurs de tous horizons : les familles, les jeunes, les "bidouilleurs" (bricoleurs et amateurs de technologies), les inventeurs et les enseignants friands de technologies. L'approche proposée vise principalement les jeunes de 8 à 14 ans, accompagnés de leurs familles, de leurs amis ou de leurs classes. Ils sont invités à imaginer une solution, la concevoir, la fabriquer et la tester : ils se trouvent au cœur de l'action!

\section{Le parcours de Fabrik}

\section{TESTER}

Choisir un défi et évaluer la solution.

Étapes : Tester et Identifier le défi

Dès que le visiteur entre dans l'exposition Fabrik, il voit des visiteurs tester leurs créations sur des bancs d'essais - de grands appareils et équipements inusités qui forment un élément visuel prédominant. Le visiteur voit les défis proposés et les solutions créées par d'autres visiteurs. Il a lui aussi envie d'inventer. C'est un déclencheur puissant. C'est donc ici, qu'il choisit son défi. C'est aussi ici, qu'il reviendra tester son prototype, la performance de sa création. C'est aussi le lieu de rencontres, de rassemblements et d'échanges avec les autres visiteurs. On observe ce qui fonctionne bien ou moins bien, et on retourne parfaire sa création. Les bancs d'essais ont été sélectionnés selon la variété d'activités qu'ils peuvent accueillir et ont été évalués auprès de jeunes. Les bancs d'essai de Fabrik sont : bac à eau, chambre noire, fil tendu en hauteur, tour de largage, test de résistance (presse, masse ou piste avec vent et réactions en chaîne). 


\section{FABRIQUER}

Concevoir et fabriquer sa solution.

Étapes : Concevoir et Fabriquer

Muni d'un défi à résoudre, le visiteur se rend à l'une des six zones de travail. Il explore une multitude de solutions, s'approvisionne en matériel, attaches et diverses composantes. Parfois, il doit déconstruire des objets pour mieux les comprendre ou se procurer des pièces importantes. Il les réorganise. Des équipements, matériaux et outils sont là pour assembler, construire, fabriquer. Ce faisant, il se familiarise avec des notions en sciences et technologies, que ce soit en optique, mécanique ou électricité. Après avoir testé sa création, il revient à cette zone afin de l'améliorer.

S'INSPIRER...

et inspirer les autres. Rassurer les visiteurs, les amener à fouiller et innover !

Étape : Partager la solution

Le visiteur, dans sa recherche de solutions, peut s'inspirer des messages et images provenant de chercheurs, inventeurs, designers et artistes, répartis sur les murs de la salle. Il y trouve des citations ; des réalisations produites par d'autres visiteurs, démontrant qu'il existe plusieurs solutions à un même problème ; une scénographie composée d'objets dont on a déformé la fonction première, un arbre-bâtons de hockeys, des lampes-roues de vélos, une paroi d'accrochage constituée de volets, autres. 


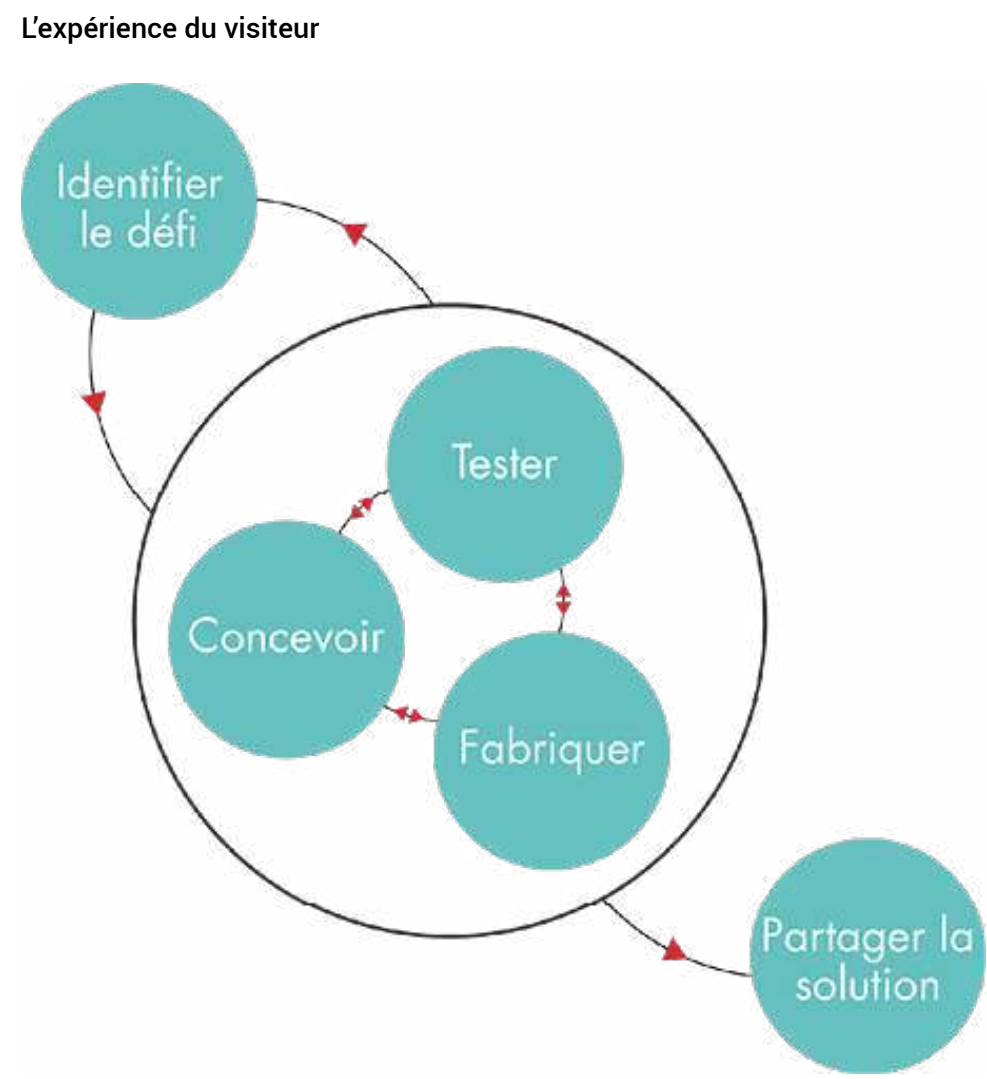

\section{Le point de vue des acteurs impliqués}

11 Selon une approche inductive, nous avons mené des entretiens non dirigés auprès de différents acteurs concernés par l'exposition Fabrik. Au Centre des sciences de Montréal, un chargé de projet et deux animateurs scientifiques nous ont donné leurs impressions et parlé de leur expérience de médiation au sein de cet équipement. Dans le milieu scolaire, une conseillère pédagogique et deux enseignants nous ont livré leurs témoignages à propos des potentialités du tinkering, tant pour leur enseignement que pour l'apprentissage de leurs élèves. D'après les informations obtenues, nous tentons de répondre à certaines questions. Comment les nouveaux lieux d'apprentissage tels l'exposition Fabrik favorisent-ils la transformation des pratiques pédagogiques en sciences et technologie ? Une exposition conçue et développée selon une approche de tinkering est-elle susceptible d'être considérée comme un nouveau lieu d'apprentissage ? Quel est le potentiel de Fabrik pour développer la culture scientifique et technique des jeunes?

\section{L'enseignement-apprentissage des sciences et de la technologie}

12 Pour plusieurs, l'enseignement des sciences et de la technologie doit suivre de manière stricte les programmes d'étude prescrits. Toutefois, certains considèrent que les programmes ne correspondent pas à leur vision "Ça [le tinkering] venait correspondre 
exactement à la vision des programmes qu'on a, au développement de compétences qu'on veut faire auprès des jeunes". Les défis proposés "apprendre aux jeunes à faire de la vraie science, ce qu'ils ne font à peu près jamais, à part quand ils arrivent à la maîtrise" semblent être en mesure de stimuler et mobiliser les élèves. "Il faut avoir un objectif, une tâche qui motive le jeune. Quand il est motivé, le jeune va mettre en pratique un paquet de compétences qu'on explore beaucoup, je pense, avec Fabrik". Le temps accordé au tinkering ne va pas de soi. "Ça peut être très insécurisant pour un enseignant de dire : je vais passer plus rapidement sur certains concepts pour pouvoir me concentrer plus sur d'autres. Il ne veut pas placer ses élèves dans l'embarras donc quand il a à faire ce choix éditorial là, il a de la difficulté à le faire".

Le tinkering, une philosophie propice aux échanges et à la coopération

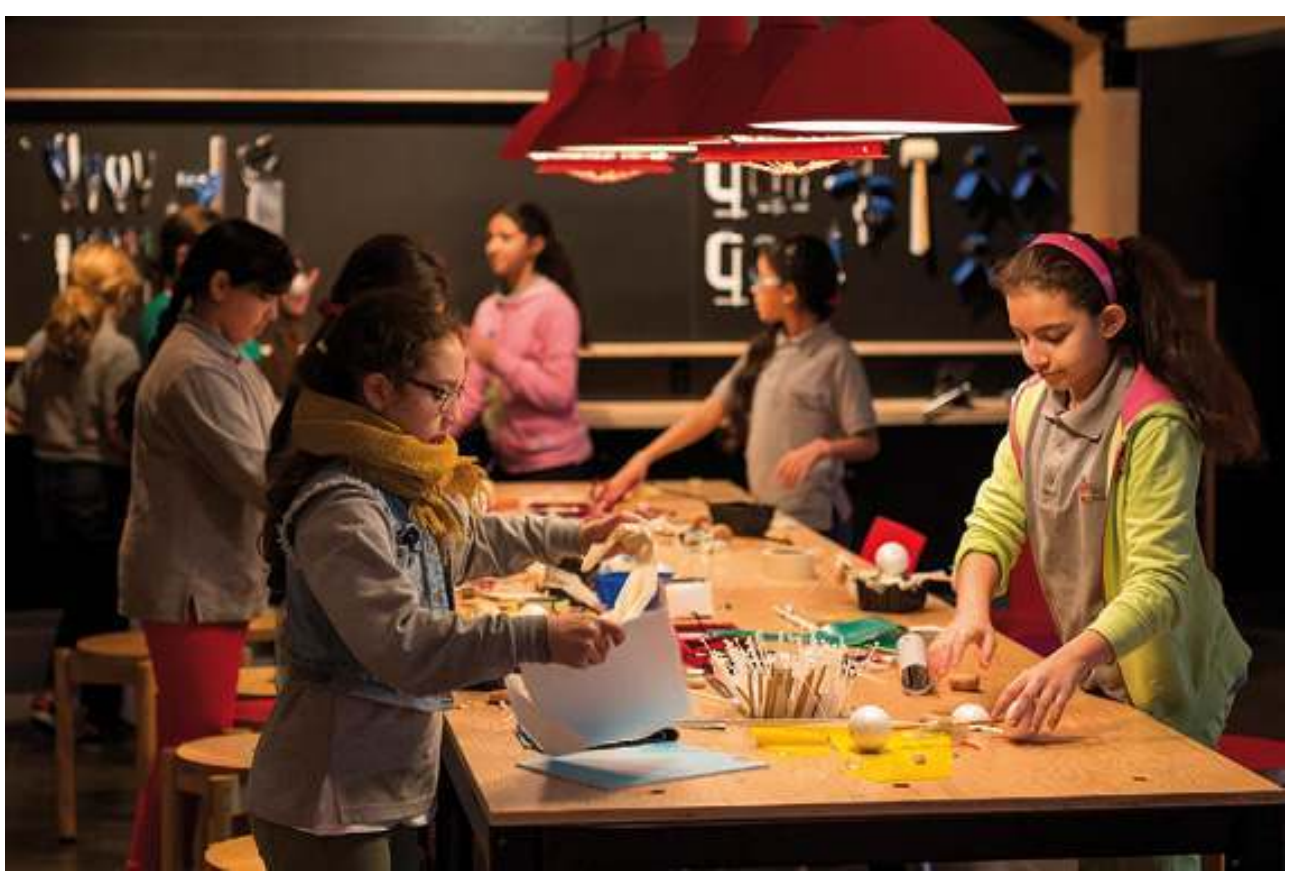

(c) CSM 


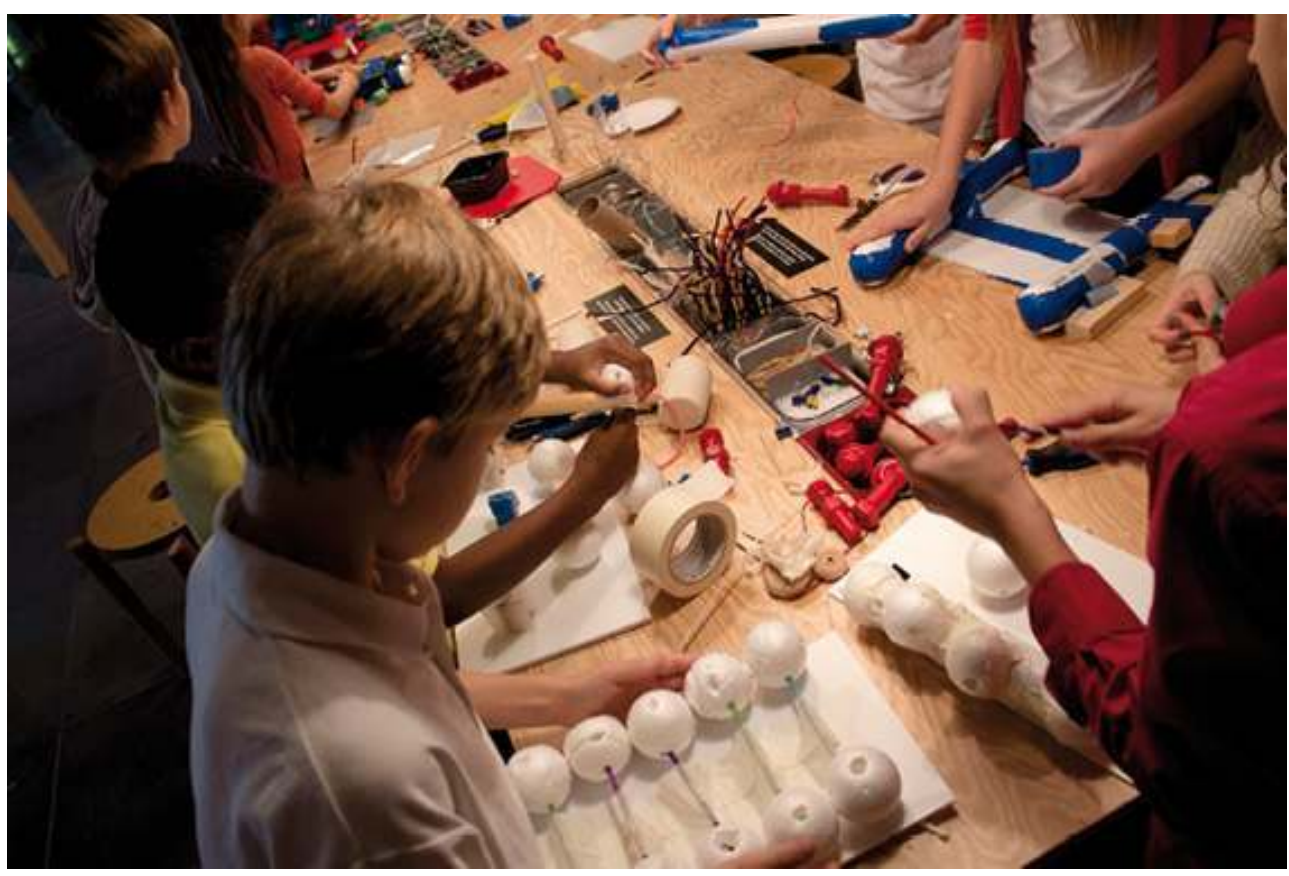

(c) CSM

Même si une grande liberté est accordée aux visiteurs dans l'exposition Fabrik, un enseignant rapporte que ses élèves se sentent très à l'aise dans cet environnement qui parait a priori "non contrôlé". Dans ce contexte propice aux échanges et à la coopération, "les élèves cohabitent, travaillent ensemble de façon très intéressante". Un lien entre l'exposition et la classe est clairement effectué par certains enseignants. "Les élèves ont pu réinvestir le travail fait au Centre des sciences dans le projet qu'ils devaient faire en classe [...] c'était une belle amorce pour moi en tant que pédagogue". Fabrik offre des opportunités en lien avec les sciences et la technologie que le milieu scolaire ne valorise pas "Ça donne des outils incroyables pour le développement de l'autonomie, la créativité, faire face à l'échec, la pensée réflexive, la rigueur mais aussi toute la motricité : interagir avec le matériel demande beaucoup de motricité, ce sont des choses qu'on oublie carrément en milieu scolaire".

\section{Vers un changement des pratiques en éducation scientifique?}

L'ouverture de Fabrik au CSM a modifié la façon dont plusieurs membres de l'équipe de médiation (concepteurs et éducateurs) perçoivent les programmes éducatifs et les expositions. "C'est une manière de penser qu'il faut changer, notre perception de comment attaquer un problème ou même un défi ou un projet, ça change". Ils se concentrent moins sur la compétition et sont plus ouverts à de nouvelles approches. "Il faut arrêter de penser à l'erreur comme une tragédie, il faut la voir comme un outil d'apprentissage". Ces constats ont inspiré une nouvelle façon de concevoir et d'animer les programmes éducatifs destinés aux groupes scolaires. "N'ayez pas peur de faire des erreurs, c'est un outil d'apprentissage très puissant". Les professionnels se concentrent davantage sur le processus que sur la quête de réussir un interactif spécifique. "On est tellement habitués, conditionnés à la bonne réponse, au succès assuré qu'on ne voit pas tous les éléments qui sont en place pour pouvoir dire: c'est un succès éducatif". Les expérimentations sont valorisées afin d'"apprendre aux jeunes à ne pas 
se gêner puis à essayer, pas avoir peur de se tromper... c'est essentiel en sciences, mais c'est essentiel dans la vie". Un participant soutient "mon idée était qu'ils soient confrontés au tinkering : ils doivent faire, refaire, améliorer, tester, prototyper rapidement. Ce que j'ai vécu comme enseignant c'est que souvent, les élèves essaient de le faire le plus parfaitement possible avant de tester". Grâce à Fabrik, il est à même d'envisager autrement "faire de la science" avec ses élèves. L'éducateur peut partager avec le groupe un bon coup, par exemple : " regardez, il a ajouté une voile, ça permet de capter plus de vent, d'augmenter la résistance de l'air et ça avance. Donner un peu de vocabulaire, mais sans aller plus loin. C'est important de donner du vocabulaire de science aux élèves du primaire". Le médiateur accompagne les jeunes dans chacune des étapes : "je vois tout le potentiel des concepts qui sont là" et facilite l'apprentissage "il faut aller chercher une réaction émotive forte, à la fois dans le négatif, parce qu'on n'a pas le choix de passer au travers, mais aussi après, dans le positif, pour marquer les jeunes. Ensuite, on va revenir sur les concepts qui vont les avoir marqués. Je pense que c'est plus payant comme ça". Dans l'exposition Fabrik, on part du concret pour placer les concepts scientifiques ensuite. Un enseignant affirme "Ce qui m'a vraiment intéressé c'est de mettre en place la dimension créative des jeunes, de faire de la science et de la techno in situ vraiment les deux mains dedans". Même discours chez les médiateurs : "L'idée c'est de lui [élève] faire découvrir avec ses mains, puis on reviendra après, à la fin ou même dans une autre période pour parler des concepts physiques qu'il a découverts par lui-même".

Les visiteurs découvrent avec leurs mains les concepts physiques.

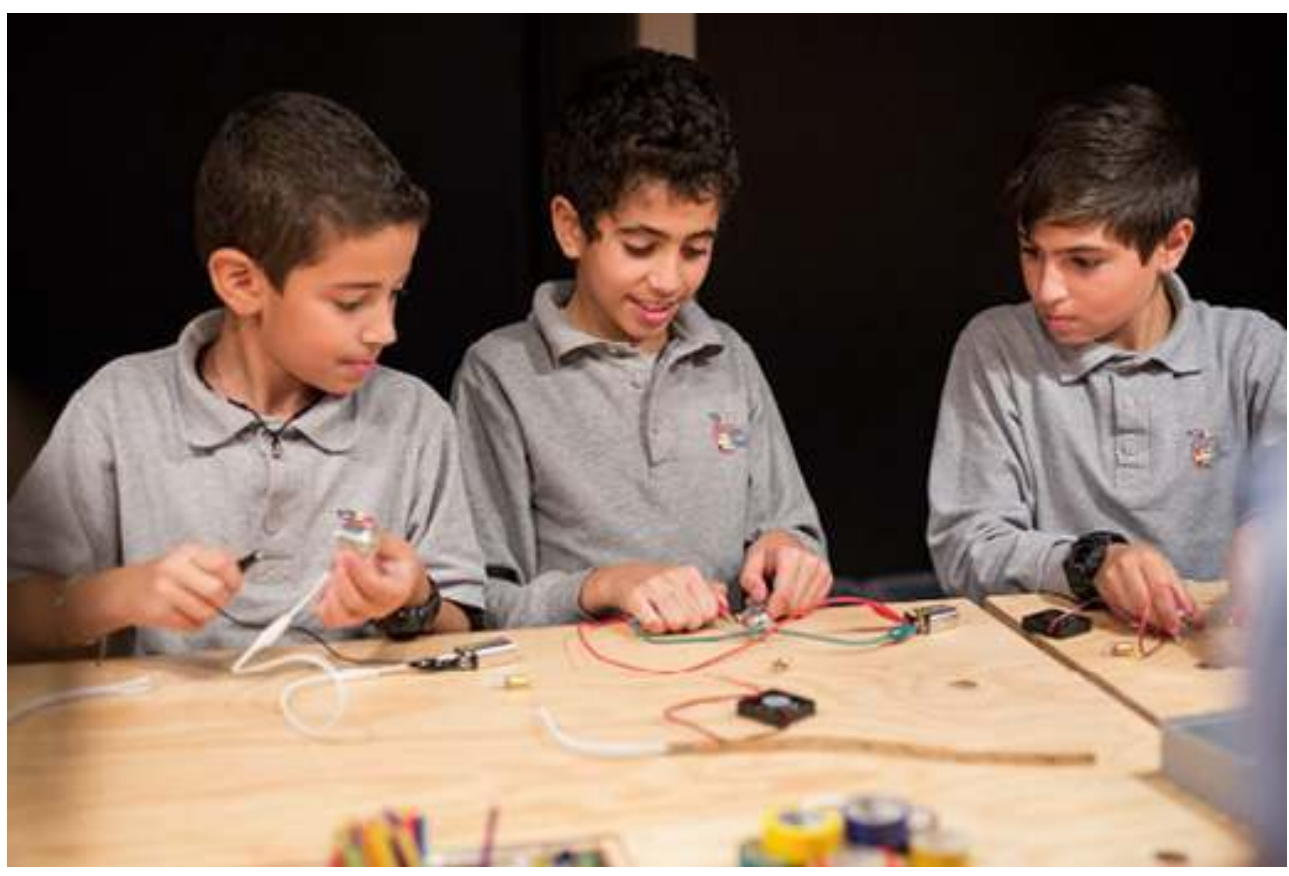

(C) CSM 


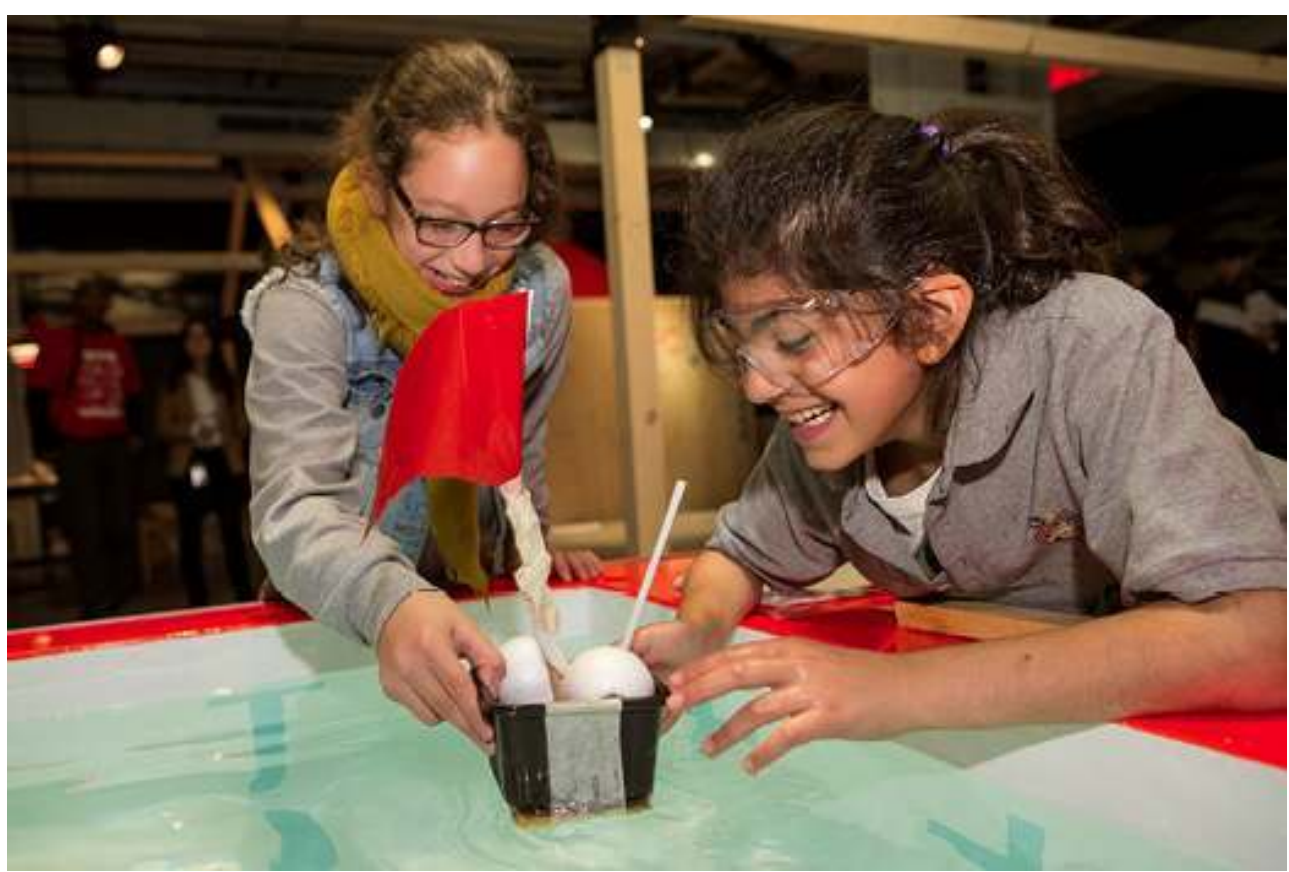

(c) CSM

Pour les enseignants, l'expérience vécue dans Fabrik comporte une rupture avec toute la tradition de l'enseignement en science en milieu scolaire. "Dans un enseignement traditionnel, l'enseignant part de son programme, va utiliser du matériel didactique déjà conçu et planifié. Dans Fabrik, c'est le contraire. On part d'une situation de la vie quotidienne, parce qu'il y a un défi qui est posé aux élèves dans une perspective d'apprentissage par problème. Il y a une redéfinition par la complexité de l'acte pédagogique parce qu'il y a une dimension improvisation qu'on ne retrouve pas dans un enseignement traditionnel". Cette modification profonde des pratiques pédagogiques implique cependant un bon nombre de freins. "C'est un changement de croyances, il faut avoir mûri et puis avoir vu le bienfait que ça peut avoir sur la réussite de nos élèves". Mais, pour l'instant tous ne sont pas prêts à se lancer dans ces nouvelles façons de faire. "Je ne les sens pas prêts, je les sens pas forts de dire : oui, je vais me lancer dans ce genre de choses-là". "Je ne pense pas que les enseignants puissent percevoir le potentiel pédagogique, l'avoir vécu fait en sorte que l'on comprend le processus à travers lequel les élèves passent et la valeur pédagogique qui s'y trouve". "Souvent, ça va servir d'initiateur". Dans des situations d'enseignement-apprentissage en classe de sciences, il n'est pas toujours aisé pour les enseignants de partir d'une réalité que tous ont pu expérimenter dans leur vie quotidienne. Un enseignant relate que pour aborder le principe de friction il ne peut se référer à la pratique du ski puisque les trois quarts de sa classe ne l'ont jamais pratiqué. Par contre, "on peut rappeler aux élèves ce qu'ils ont vécu dans Fabrik pour qu'ils comprennent mieux ce qui est abordé en classe. C'est une façon de s'assurer que tout le monde a le même contexte, la même base et qu'ils ont tous déjà été confrontés à ces phénomènes. Cela les ramène à une expérience vécue et à laquelle ils peuvent rattacher un concept scientifique qui leur permet d'aller plus loin". Pour certains enseignants, ce type d'approche ne correspond pas à leur style d'enseignement, ils ont besoin de rester en contrôle de la situation pédagogique. Le tinkering n'est donc certainement pas une approche à privilégier pour eux. Toutefois, cela peut très bien correspondre à d'autres. "On réutilise des situation ayant eu lieu dans Fabrik, en classe. C'est un élément intégré à ma planification de projet". "S'il n'y avait plus Fabrik au Centre des sciences, j'essaierais de proposer à mes élèves des défis qui les mettraient dans le 
même mode de pensée, car je trouve cela intéressant". Enfin, des enseignants ont modifié leurs pratiques pédagogiques en lien avec les expériences vécues au CSM. "Depuis que je connais Fabrik, ça a contaminé la façon dont j'enseigne. Je donne désormais un objectif final précis à atteindre et laisse plus de latitude dans l'accomplissement du projet. Cela a réduit l'aspect uniforme et semblable des projets des élèves".

\section{Des rôles qui se redéfinissent}

Dans les programmes d'étude actuels, l'enseignant ne possède plus l'unique rôle de détenteur et transmetteur du savoir. Il est davantage perçu comme un "médiateur" ou un "passeur culturel". Selon cette même perspective, dans Fabrik, "l'enseignant devient plutôt un médiateur entre le savoir et ce que l'enfant doit apprendre". Certains enseignants se laissent même prendre au jeu et "le fait de se placer dans une position d'apprenant avec les élèves permet de leur montrer que même si on est leur enseignant, dans un autre contexte on retourne au rôle d'apprenant". Ce rôle d'apprenant endossé tant par les élèves que leur enseignant appelle une posture d'humilité lorsque l'apprenti dépasse le maître. "Ça laisse place à des résultats originaux auxquels l'enseignant ne pense pas forcément, parfois les résultats des élèves sont meilleurs que les nôtres". Dans l'exposition, le médiateur apprend à observer les visiteurs et à décoder les signes de blocage ou de découragement. Autant que possible, il ne doit pas donner de réponses, mais conseiller et relancer les participants : "Ta solution est excellente. Bravo! Toutefois, elle utilise beaucoup de matériaux. Peux-tu en trouver une autre tout aussi efficace en utilisant moins de matériaux ?" Le rôle des éducateurs est de laisser beaucoup de liberté aux jeunes afin qu'ils puissent expérimenter par eux-mêmes. Par contre, ils doivent être présents pour procurer de l'aide et soutenir les visiteurs dans leur démarche de création. "À Fabrik, on fonctionne à l'envers parce que ce sont les jeunes qui vont découvrir la science, ce sont eux qui doivent manipuler puis, on doit les guider dans cette découverte-là. On doit passer d'un rôle de démonstrateur à un rôle d'accompagnateur". En posant des questions comme "d'après toi, pourquoi ça ne marche pas ?", il s'agit de "créer une ambiance pour pousser encore plus loin les apprentissages".

\section{Les lendemains du tinkering}

17 "Ça a été un coup de cour dès le départ cette exposition là !" Plusieurs personnes, notamment des enseignants et des médiateurs ne peuvent cacher leur enthousiasme pour les approches de tinkering comme celle qui est offerte par l'exposition Fabrik du Centre des sciences de Montréal. Elles apportent un souffle renouvelé à l'éducation scientifique en proposant des espaces non conventionnels où les visiteurs sont appelés à penser autrement et peut-être même apprendre autrement. "Il faudrait essayer de toucher d'autres domaines que seulement la physique et l'ingénierie, j'ai en tête un Fabrik d'ambulancier ou de premiers soins, par exemple". Toutefois, nous ne pouvons généraliser cet engouement, ni passer sous silence le débat qui fait rage dans la communauté de la muséologie scientifique : les ateliers de tinkering ont-ils vraiment leur place dans les centres de sciences? Certaines personnes persistent à croire qu'on n'y apprend guère de nouvelles connaissances. Est-ce là la mission légitimée des équipements de culture scientifique et technologique ? L'éveil des sens et de l'esprit n'aurait-il pas sa place dans les centres de sciences ? L'apprentissage de méthodes, d'intuition de fabrication et conception ne devrait-il pas aussi être au centre d'une programmation en éducation technoscientifique 
? Pourtant, un enseignant insiste "la valeur pédagogique que j'associe à ce qu'on trouve dans Fabrik est suffisamment importante pour qu'on envisage le refaire en classe".

Les auteurs remercient Julie Rose, étudiante à la maîtrise en muséologie de l'université du Québec à Montréal, pour sa participation au travail de collecte et d'analyse des données.

\section{RÉSUMÉS}

L'espace Fabrik du Centre de sciences de Montréal s'est donné comme objectif de développer chez les visiteurs, en particulier chez les plus jeunes, la créativité, l'inventivité et l'autonomie grâce à la manipulation d'outils, de matériaux et de technologies. Les auteurs montrent que cette manière d'apprendre "en faisant" bouleverse les pratiques de l'éducation scientifique tant en contexte muséal que scolaire.

\section{INDEX}

Mots-clés : Fab Lab, réseau, CSTI

\section{AUTEURS}

\section{ANIK MEUNIER}

Professeure titulaire et chercheure en éducation et muséologie, directrice du Groupe de recherche sur l'éducation et les musées, Université du Québec à Montréal meunier.anik@uqam.ca

\section{BRIGITTE BELLEVILLE}

Chargée de projets, développement et réalisation, Centre des sciences de Montréal, conceptrice de l'exposition Fabrik

bbelleville@vieuxportdemontreal.com

\section{OLIVIER GRANT}

Chef éducation, Centre des sciences de Montréal, concepteur de l'exposition Fabrik ogrant@vieuxportdemontreal.com 